\title{
Using automated pump-delivery devices to reduce the incidence of excessive fluid administration during pediatric dental surgery: a randomized-controlled trial
}

\section{L'impact des pompes pour réduire l'incidence d'administration liquidienne excessive pendant une chirurgie dentaire pédiatrique : une étude randomisée contrôlée}

\author{
Duncan E. Bowes, MD (1) • Jonathan J. Gamble, MD • Jagmeet S. Bajwa, MSc
}

Received: 2 February 2020/Revised: 22 May 2020/Accepted: 23 May 2020/Published online: 5 August 2020

(C) Canadian Anesthesiologists' Society 2020

\begin{abstract}
Purpose The harms caused by excessive perioperative intravenous (IV) fluid administration are both well recognized and avoidable. The purpose of this study was to compare the incidence of excess intraoperative fluid administration in pediatric dental surgery patients when either an automated pump-delivery device or a manual gravity-drip device is used.

Methods We randomly assigned American Society of Anesthesiologists physical status I and II pediatric dental surgery patients to receive IV fluid via either a manual gravity-drip or automated pump-delivery device. Prior to each case, the attending anesthesiologist determined the target volume of maintenance IV fluid to be administered based on patient weight, estimated fluid deficits, and expected case length. The intraoperative IV fluid delivered was determined at the end of the case by the change in the $I V$ bag weight. The primary outcome was the proportion of procedures that delivered $\geq 10 \%$ of the target IV fluid volume.

Results: We recruited 105 children aged two to $12 \mathrm{yr}(n=$ 49 in the automated pump-delivery device; $n=53$ in the manual gravity-drip device). The proportion of excessive fluid administration was $8 / 49(16 \%)$ in the automated pump-delivery device group compared with 33/53 (62\%) in the gravity-drip group (relative risk of excessive fluid
\end{abstract}

D. E. Bowes, MD (凹) · J. J. Gamble, MD · J. S. Bajwa, MSc Department of Anesthesiology, Perioperative Medicine and Pain Management, Royal University Hospital, University of

Saskatchewan, G525-103 Hospital Drive, Saskatoon, SK S7N 0W8, Canada

e-mail: Duncan.Bowes@usask.ca administration, $0.26 ; 95 \%$ confidence interval, 0.13 to $0.51 ; P<0.001)$.

Conclusion Intraoperative fluid administration using an automated pump-delivery device decreased the incidence of excessive IV fluid administration in pediatric dental surgery patients.

Trial registration www.clinicaltrials.gov (NCT033124 52); registered 17 October 2017.

\section{Résumé}

Objectif Les effets néfastes causés par une administration liquidienne intraveineuse (IV) périopératoire excessive sont à la fois bien connus et évitables. L'objectif de cette étude était de comparer l'incidence d'administration liquidienne peropératoire excessive chez les patients pédiatriques de chirurgie dentaire lors de l'utilisation d'une pompe à perfusion automatique vs un système goutte à goutte manuel par gravité.

Méthode Nous avons randomisé des patients pédiatriques de chirurgie dentaire de statut physique I et II selon l'American Society of Anesthesiologists à recevoir des liquides intraveineux soit par un système goutte à goutte manuel par gravité ou via une pompe à perfusion automatique. Avant chaque cas, l'anesthésiologiste en charge a déterminé le volume cible de liquide IV de maintien à administrer selon le poids du patient, les déficits liquidiens estimés, et la durée anticipée du cas. La quantité de liquides IV peropératoires administrés était déterminée à la fin du cas par le changement du poids du sac de liquide IV. Le critère d'évaluation principal était la proportion d'interventions ayant administré $\geq 10 \%$ du volume liquidien IV cible. 
Résultats Nous avons recruté 105 enfants âgés de deux à 12 ans ( $n=49$ dans le groupe pompe automatique; $n=53$ dans le groupe goutte à goutte manuel). La proportion d'administration liquidienne excessive était de 8/49 (16\%) dans le groupe pompe automatique, comparativement à 33/ $53(62 \%)$ dans le groupe goutte à goutte (risque relatif d'administration liquidienne excessive, 0,26; intervalle de confiance $95 \%, 0,13$ à 0,51; $P<0,001$ ).

Conclusion L'administration liquidienne peropératoire à l'aide d'une pompe a réduit l'incidence d'administration liquidienne IV excessive chez des patients pédiatriques de chirurgie dentaire.

Enregistrement de l'étude ww.clinicaltrials.gov (NCT03 312452); enregistrée le 17 octobre 2017.

Keywords Child · random allocation · anesthesiology · prospective studies $\cdot$ automated pump-delivery device

Excessive intravenous (IV) fluid administration can lead to electrolyte abnormalities, ${ }^{1,2}$ prolonged mechanical ventilation, ${ }^{3}$ acute kidney injury, ${ }^{4}$ neurologic injury, ${ }^{2}$ and mortality. ${ }^{3,5,6}$ Pediatric patients may be more susceptible to some of these iatrogenic harms. ${ }^{2}$ To mitigate these risks, IV fluids should be administered with the same precision afforded to other drugs. ${ }^{7}$

Errors in IV fluid administration are common and account for almost $80 \%$ of reported in-hospital medication errors. ${ }^{8}$ Although manual gravity-drip devices are frequently used during intraoperative care, they are often imprecise. ${ }^{9,10}$ The rate of fluid administration by a conventional micro-drip device can vary nearly three-fold depending on the fluid column height and size of the IV catheter. ${ }^{10} \mathrm{~A}$ study of hospitalized patients reported only $26 \%$ of continuous IV fluid infusions were administered at the target rate when delivered by a gravity-drip device. ${ }^{11}$ This study further suggested a near $90 \%$ odds reduction of fluid administration error when an automated pumpdelivery device is used instead of a gravity-drip device. ${ }^{11}$

Given the risks associated with excessive intraoperative fluid administration in pediatric patients and the lack of research comparing automated devices with manual devices, we hypothesized an automated pump-delivery device would reduce the incidence of excessive intraoperative fluid administration compared with a manual gravity-drip device.

\section{Methods}

The study protocol was approved by the Biomedical Research Ethics Board of the University of Saskatchewan
(30 August 2017). The study protocol was registered on 17 October 2017 at clinicaltrials.gov (NCT03312452).

Healthy children (American Society of Anesthesiologists [ASA] physical status I and II) aged 2$12 \mathrm{yr}$, undergoing dental surgery at an ambulatory surgical centre between August 27 and October 212018 were eligible for inclusion. Written informed parental/legal guardian consent and participant assent were obtained preoperatively.

\section{Experimental protocol}

This blinded clinical trial randomly allocated patients in a 1:1 ratio into two groups based on the device used to control IV fluid rate, either an automated pump-delivery device (Plum A+ infusion pump; Hospira, Lake Forest, IL, USA) or a manual gravity-drip device (60 drops. $\mathrm{mL}^{-1}$ micro-drip primary set; ICU Medical Inc., San Clemente, CA, USA). Randomization was implemented with the online random number generator (Randomness and Integrity Services, Dublin, Ireland).

Prior to each procedure, the attending anesthesiologist independently determined a target IV fluid volume to be administered after considering the patient's potential fluid deficit, weight, and case duration as estimated by the surgeon. At our institution, for ambulatory pediatric anesthesia procedures, some anesthesiologists elect to administer a fluid bolus at the start of a case. ${ }^{12}$ This volume is provider specific. Patients assigned to the automated pump-delivery device received IV fluid at a rate equaling (target volume + planned bolus volume)/ estimated case length. For those cases with a planned fluid bolus, the automated pump-delivery device was programmed to administer the bolus volume at the pump's maximum rate $\left(999 \mathrm{~mL} \cdot \mathrm{hr}^{-1}\right)$, then reprogrammed to the target rate described above. Patients assigned to the manual gravity-drip device received all planned IV fluid at the discretion of the attending anesthesiologist. Unanticipated intraoperative fluid boluses were administered at the clinician's discretion and the volume of these boluses were subtracted from the final measured volume. All unplanned, intraoperative boluses were reported to the investigator and recorded.

Data collection and outcomes

Demographic data included patient weight, length of preoperative fasting (minutes), sex, dentist estimated length of case (hours), and ASA physical status. The primary outcome was the proportion of procedures that delivered $\geq 10 \%$ of the target IV fluid volume. Excessive fluid administration is a widely recognized phenomenon with little diagnostic consensus. ${ }^{3}$ In a study of fluid 


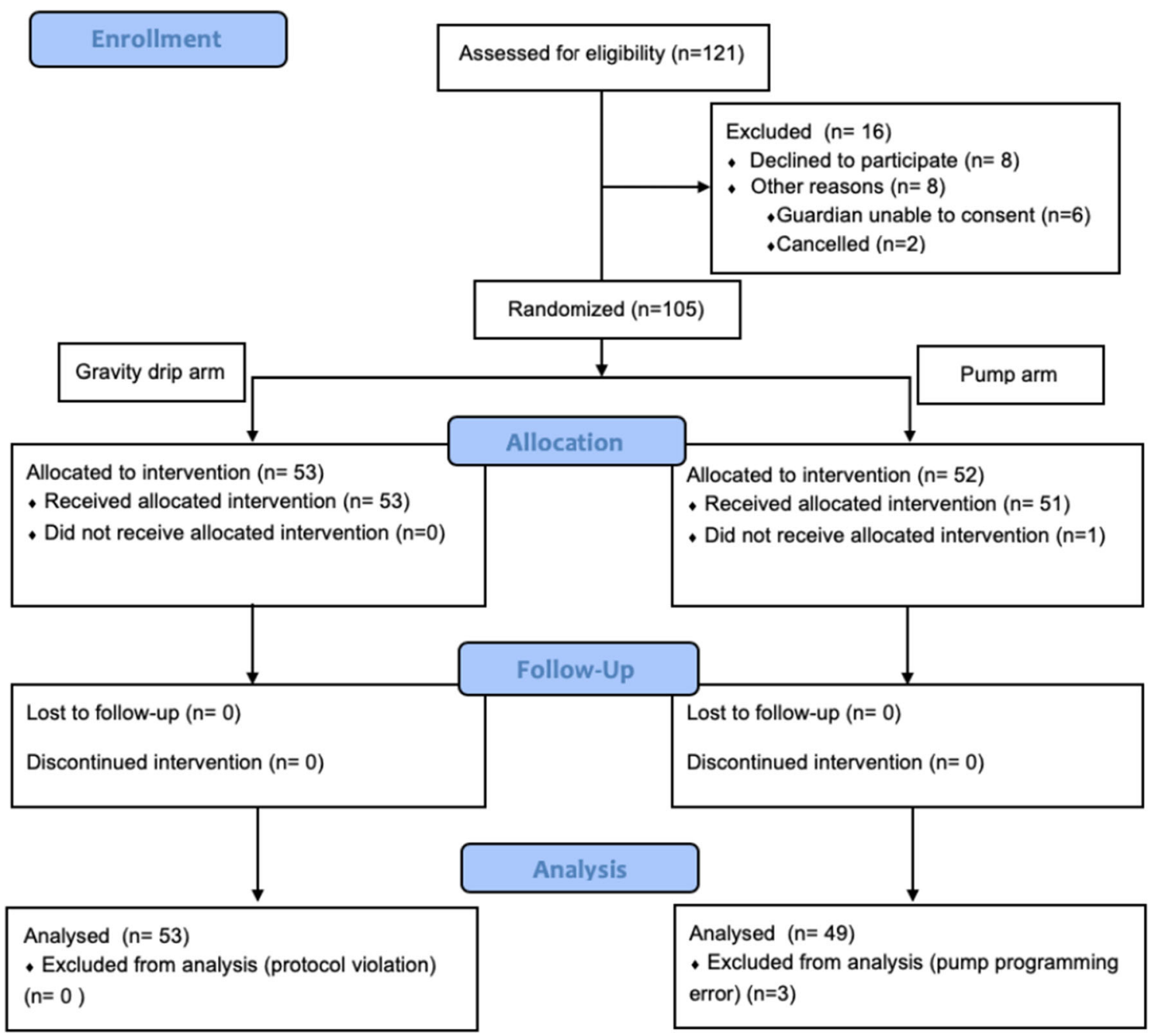

FIGURE CONSORT flow diagram.

infusion rates in medical inpatients, an accurate rate of administration was defined as being within $0-10 \%$ of the provider-prescribed target rate. ${ }^{11}$ Secondary outcomes included IV fluid prescribed and administered, and the difference between the two (all in $\mathrm{mL} \cdot \mathrm{kg}^{-1}$ ). Prior to each procedure, a standard $500 \mathrm{~mL}$ bag of Ringer's lactate (Baxter, Deerfield, IL, USA) was weighed in triplicate to obtain a mean pre-procedure weight. At the end of each case, the used IV fluid bag was again weighed in triplicate to obtain a mean post-procedure weight. The final volume of fluid administered was calculated by dividing the difference in mean pre- and post-procedure IV bag weight by the known density of Ringer's lactate (1.01 $\mathrm{g} \cdot \mathrm{mL}^{-1}$ ). To account for the fluid in the IV tubing at the end of the procedure, a manufacturer specified volume was subtracted from the final calculated volume of each IV fluid bag $(14 \mathrm{~mL}$ in the manual gravity-drip arm, $19 \mathrm{~mL}$ in the automated pump-delivery device arm). As maintenance fluid volume varies according to procedure duration, we defined the procedure duration as the time from the initiation of IV access until the anesthesiologist fluid locked the patient's IV (it is standard practice at our institution to fluid lock the IV at the end the procedure prior to transfer to the postanesthesia care unit). Administered volumes were standardized by time to account for the effect of surgery duration. Transition to the postanesthesia care unit was the end of patient follow-up.

Sample size was based on the estimate that the proportion of cases with excessive fluid administration would be 0.4 in the manual gravity-drip group and 0.1 in the automated pump-delivery device group. Estimates were entered into an online sample size calculator (Statulator, Sydney, Australia) to produce an estimated sample size of 72 participants (36 participants in each arm). To account for the usual dropouts and technical problems, a total sample size of 96 participants was chosen. 
Statistical analysis

Statistical analysis was performed with IBM SPSS Statistics version 25.0 (Armonk, NY, USA) by a statistician blinded to patient allocation. The primary outcome (proportion of cases that delivered $\geq 10 \%$ of the target IV fluid volume) was compared with a PearsonChi square test. Relative risk and $95 \%$ confidence intervals (CI) were calculated. Secondary outcomes (prescribed and administered IV fluid, and the difference between the two) were compared with a Student's $t$ test. A two-tailed level of significance was set at 0.05 . All outcomes were analyzed using intention-to-treat analysis.

\section{Results}

One hundred and twenty-one patients were approached to participate with 53 and 52 well matched patients allocated to automated pump-delivery device group and manual gravity-drip groups, respectively (Figure and Table 1). Because of an administrative oversight, an additional nine patients were recruited beyond our original target of 96.

In total, 33/53 (62\%) children randomized to the manual gravity-drip arm, and $8 / 49(16 \%)$ randomized to the automated pump-delivery device group received a volume of fluid $\geq 10 \%$ of the target. The relative risk of excessive IV fluid administration with an automated pumpdelivery device was 0.26 (95\% CI, 0.13 to $0.51 ; P<$ $0.001)$. Compared with children allocated to an automated pump-delivery device, children in the manual gravity-drip arm received $3.2 \mathrm{~mL} \cdot \mathrm{kg}^{-1}$ more fluid than their initial target volume (95\% CI, 1.29 to $5.17 ; P<0.001)$, (Table 2 ). Unanticipated fluid boluses were infrequent (1/53 and 4/49 in the gravity-drip and automated pump-delivery device arms, respectively) and did not account for excessive IV fluid administration (Table 2). Anesthesiologists' fluid prescriptive practices were similar between groups (15.8 $\mathrm{mL} \cdot \mathrm{kg}^{-1}$ in the manual gravity-drip group $v s 17.8 \mathrm{~mL} \cdot \mathrm{kg}^{-1}$ in the automated pump-delivery device group; variance ratio, $1.6 ; 95 \% \mathrm{CI}, 0.91$ to $2.79 ; P=0.06)$.

\section{Discussion}

This randomized-controlled trial showed excessive IV fluid administration occurs significantly more often when IV fluid volume is controlled using a manual gravity-drip device compared with an automated pump-delivery device in pediatric dental surgery. Previously published research has investigated perioperative restrictive and liberal fluid administration practices, but studies quantifying intraoperative errors in fluid administration are lacking. ${ }^{13,14}$ Cross-sectional studies in adult inpatients have reported errors in IV fluid administration rates ranging from $18 \%$ to $74 \% .^{8,11}$ Our total excessive fluid administration rate (both arms) of $40 \%$ is consistent with these studies. In contrast to our findings, the majority of reported administration errors were due to fluid underadministration. In our study, only 12 of 102 total cases (12\%) received less fluid than targeted, with excessive administration occurring much more frequently. In keeping with our findings, automated pump-delivery devices have consistently shown improved precision in hospital inpatients. ${ }^{11}$ A study of medical inpatients reported an

Table 1 Patient and surgical case characteristics

\begin{tabular}{|c|c|c|}
\hline Characteristic & $\begin{array}{l}\text { Gravity-drip device } \\
n=53\end{array}$ & $\begin{array}{l}\text { Automated pump-delivery device } \\
n=49\end{array}$ \\
\hline Male, $n /$ total $n(\%)$ & $27 / 53(51)$ & $24 / 49$ (49) \\
\hline Age (yr) & $4.9(2.2)$ & $5(2.4)$ \\
\hline Weight (kg) & $20.8(5.6)$ & $21.6(8.5)$ \\
\hline ASA physical status & $42 / 53(79)$ & $40 / 49(82)$ \\
\hline Healthy, $n /$ total $n(\%)$ & $11 / 53(21)$ & $9 / 49(18)$ \\
\hline \multicolumn{3}{|c|}{ Mild systemic disease, $n /$ total $n(\%)$} \\
\hline Length of fast (min) & 779 (122) & $782(161)$ \\
\hline Case booked for (hr) & $1.6(0.3)$ & $1.6(0.3)$ \\
\hline Length of case, IV PACU (hr) & $1.3(0.4)$ & $1.3(0.4)$ \\
\hline Preop weight of bag (g) & $572(2)$ & $572(2)$ \\
\hline
\end{tabular}

Data are presented as means with standard deviation in parenthesis unless otherwise stated.

ASA = American Society of Anesthesiologists; IV = intravenous; PACU = postanesthesia care unit. 
Table 2 Primary and secondary study outcomes with associated relative risks

\begin{tabular}{|c|c|c|c|c|}
\hline Outcome & $\begin{array}{l}\text { Gravity-driven } \\
\text { devices } \\
n=53\end{array}$ & $\begin{array}{l}\text { Automated } \\
\text { pump-delivery device } \\
n=49\end{array}$ & $\begin{array}{l}\text { Relative risk } \\
(95 \% \mathrm{CI})\end{array}$ & $P$ value \\
\hline \multicolumn{5}{|l|}{ Primary outcome } \\
\hline \multicolumn{5}{|l|}{ (Yes), $n /$ total $n(\%)$} \\
\hline \multicolumn{5}{|l|}{ Continuous secondary outcomes } \\
\hline & & & Variance ratio $(95 \% \mathrm{CI})$ & \\
\hline Planned fluid prescribed $\left(\mathrm{mL} \cdot \mathrm{kg}^{-1}\right.$ mean $(\mathrm{SD})$ & $15.8(8.1)$ & $17.8(6.4)$ & $1.6(0.91$ to 2.79$)$ & $0.06^{\mathrm{c}}$ \\
\hline Planned fluid administered $\left(\mathrm{mL} \cdot \mathrm{kg}^{-1}\right)$, mean (SD) & $16.3(7.1)$ & $15.1(6.3)$ & $1.3(0.72$ to 2.21$)$ & $0.48^{\mathrm{c}}$ \\
\hline \multirow{2}{*}{$\begin{array}{l}\text { Total (including unplanned boluses) fluid } \\
\text { administered }\left(\mathrm{mL} \cdot \mathrm{kg}^{-1}\right) \text {, mean (SD) }\end{array}$} & $16.5(7.1)$ & $15.2(6.4)$ & $1.2(0.69$ to 2.15$)$ & $0.48^{\mathrm{c}}$ \\
\hline & & & $\begin{array}{l}\text { Mean difference } \\
(95 \% \text { CI })\end{array}$ & $\begin{array}{l}<0.001^{\mathrm{b}} \\
<0.001^{\mathrm{b}}\end{array}$ \\
\hline $\begin{array}{l}\text { Difference between fluid prescribed and administered } \\
\left(\mathrm{mL} \cdot \mathrm{kg}^{-1}\right) \text {, planned fluid }\end{array}$ & $0.52(5.8)$ & $-2.7(3.7)$ & $3.2(1.29$ to 5.17$)$ & \\
\hline $\begin{array}{l}\text { Difference between fluid prescribed and administered } \\
\left(\mathrm{mL} \cdot \mathrm{kg}^{-1}\right) \text {, total fluid (including unplanned boluses) }\end{array}$ & $0.70(5.8)$ & $-2.5(3.8)$ & $3.2(1.32$ to 5.19$)$ & \\
\hline
\end{tabular}

a Pearson-Chi square test ${ }^{\mathrm{b}}$ Student's $t$ test ${ }^{\mathrm{c}}$ Levene test. CI = confidence interval; IV = intravenous; SD = standard deviation.

$18 \%$ absolute increase in the precision of IV fluid administration rates while another study found an $88 \%$ reduction in the odds of an administration error. ${ }^{8}$ Our study suggests that clinicians are more than twice as likely to precisely administer the target IV fluid volume when using a automated pump-delivery device compared with using gravity-drip devices (associated relative risk, 0.46; 95\% CI, 0.29 to 0.63 ).

The aim of this study was to determine if excessive IV fluid administration could be reduced by the use of an automated pump-delivery device compared with a manual gravity-drip device in pediatric patients. Our intent was not to investigate clinical benefit given the low risk nature of the procedure and study population. A difference of 3.2 $\mathrm{mL} \cdot \mathrm{kg}^{-1}$ of fluid in otherwise healthy patients is of questionable clinical significance. Nevertheless, it is reasonable to suspect similar findings would be observed in children at greater risk of harm due to excessive fluid administration, such as those with major comorbidities presenting for more invasive procedures when gravity-drip devices are used.

Pediatric patients may be more susceptible to iatrogenic harms related to medication administration errors. ${ }^{15,16}$ Additionally, pediatric fluid overload remains a common clinical problem; in a systematic review of pediatric patients admitted to the intensive care unit, the odds of mortality increased by $6 \%$ for every $1 \%$ increase in fluid balance. ${ }^{3,17}$ The burden of harm associated with excessive fluid administration is not exclusive to critically ill children; several case reports detail harms suffered by otherwise healthy patients presenting for elective surgery. ${ }^{1,2,18}$ Our study shows how frequently clinicians unintentionally administer excessive fluid during routine clinical care. Although our study shows more precise fluid administration when automated delivery devices are used, this technology does not completely eliminate the potential for error. ${ }^{19}$ It has been reported that almost $10 \%$ of all observed drug administration errors were related to discrepancies between prescription and programing. All but one of these errors were judged as having been unlikely to be prevented by an automated device's technology. ${ }^{19}$

The strengths of this study include the study design (randomized clinical trial), which minimized the risk of bias due to unmeasured confounders. The study groups were well matched with respect to baseline characteristics (Table 1), operative case time, and anesthesiologist IV fluid prescription practices (Table 2). Limitations included our inability to blind the attending anesthesiologists to patient allocation on account of the additional noise produced by the automated pump-delivery device. This may have led to a Hawthorne effect and increased vigilance to fluid administration amongst the attending anesthesiologists. All cases used standardized $500 \mathrm{~mL}$ bags of Ringer's lactate, which may have served as an additional cue to the attending clinician to restrict subsequent fluid administration, especially if an additional bag of fluid was required. It seems likely that both of these phenomena would be mitigated in the automated pump-delivery device arm suggesting our 
results underestimate the risk of excessive fluid administration.

\section{Conclusions}

Intravenous fluid delivery using an automated pumpdelivery device reduces the rate of excessive fluid administration compared with using a manual gravitydrip device in the setting of ambulatory pediatric anesthesia. Future studies should investigate how intraoperative fluid control devices affect clinical outcomes.

Author contributions Duncan Bowes and John Gamble contributed to all aspects of this manuscript, including study conception and design; acquisition, analysis, and interpretation of data; and drafting the article. Jagmeet Bajwa contributed to the analysis of data.

Acknowledgements The trial investigators are grateful to the University of Saskatchewan's College of Medicine and Department of Anesthesiology, Perioperative Medicine and Pain Management for funding this study.

Disclosures None.

Funding statement University of Saskatchewan, College of Medicine Research Award (CoMRAD).

Editorial responsibility This submission was handled by Dr. Hilary P. Grocott, Editor-in-Chief, Canadian Journal of Anesthesia.

\section{References}

1. Koczmara C, Hyland S, Greenall J. Hospital-acquired acute hyponatremia and parenteral fluid administration in children. Can J Hosp Pharm 2009; 62: 512-5.

2. Moritz ML, Ayus JC. Prevention of hospital-acquired hyponatremia: a case for using isotonic saline. J Pediatr 2003; 11: 227-30.

3. Alobaidi R, Morgan C, Basu R, et al. Association between fluid balance and outcomes in critically ill children. a systematic review and meta-analysis. JAMA Pediatr 2018; 172: 257-68.
4. Li Y, Wang J, Bai Z, et al. Early fluid overload is associated with acute kidney injury and PICU mortality in critically ill children. Eur J Pediatr 2016; 175: 39-48.

5. Maitland K, Kiguli S, Opoka RO, et al. Mortality after fluid bolus in African children with severe infection. N Engl J Med 2011; 22: 2483-95.

6. Cunliffe M, Potter F. Four and a fifth and all that. Br J Anaesth 2006; 97: 274-7.

7. Feld LG, Neuspiel DR, Foster BA, et al. Clinical practice guideline: maintenance intravenous fluids in children. J Pediatr 2018; . https://doi.org/10.1542/peds.2018-3083.

8. Han PY, Coombes ID, Green B. Factors predictive of intravenous fluid administration errors in Australian surgical care wards. Qual Saf Health Care 2005; 14: 179-84.

9. Pico LJ. An appliance to facilitate the administration of intravenous anesthesia by continuous drip. Anesthesiology 1944; 5: 411.

10. Pierce ET, Kumar V, Zheng H, Peterfreund RA. Medication and volume delivery by gravity-driven micro-drip intravenous infusion: potential variations during "wide-open" flow. Anesth Analg 2013; 116: 614-8.

11. Rooker JC, Gorard DA. Errors of intravenous fluid infusion rates in medical inpatients. Clin Med (Lond) 2007; 7: 482-5.

12. Gan TJ, Diemunsch P, Habib AS, et al. Consensus guidelines for the management of postoperative nausea and vomiting. Anesth Analg 2014; 118: 85-113

13. Myles PS, Bellomo R, Corcoran T, et al. Restrictive versus liberal fluid therapy for major abdominal surgery. N Engl J Med 2018; 328: 2263-74.

14. Nisanevich V, Felsenstein I, Almogy G, Weissman C, Einav S, Matot I. Effect of intraoperative fluid management on outcome after intraabdominal surgery. Anesthesiology 2005; 103: 25-32.

15. Fernandez $C V$, Gillis-Ring J. Strategies for the prevention of medical error in pediatrics. J Pediatr 2003; 143: 155-62.

16. Kaushal R, Bates DW, Landrigan C, et al. Medication errors and adverse drug events in pediatric inpatients. JAMA 2001; 285: 2114-20.

17. Hassinger $A B$, Valentine SL. Self-reported management of iv fluids and fluid accumulation in children with acute respiratory failure. Pediatr Crit Care Med 2018; 19: e551-4.

18. Arieff AI, Ayus JC, Fraser CL. Hyponatraemia and death or permanent brain damage in healthy children. BMJ 1992; 304: 1218-22.

19. Husch $M$, Sullivan $C$, Rooney $D$, et al. Insights from the sharp end of intravenous medication errors: implications for infusion pump technology. Qual Saf Health Care 2005; 14: 80-6.

Publisher's Note Springer Nature remains neutral with regard to jurisdictional claims in published maps and institutional affiliations. 